\title{
Aspectos clínicos y tratamiento endoscópico de la hemorragia digestiva por lesión de Dieulafoy
}

\author{
A. Ibañez, E. Castro, E. Fernández, R. Baltar, S. Vázquez, J. L. Ulla, V. Álvarez, S. Soto, J. Barrio, \\ D. Carpio, J. Turnes, L. Ledo, J. Vázquez San Luis y E. Vázquez Astray
}

Servicio de Aparato Digestivo. Complejo Hospitalario de Pontevedra. Hospital Montecelo. Pontevedra

\section{RESUMEN}

Objetivo: conocer la incidencia, forma de presentación, localización y resultados del tratamiento endoscópico en la hemorragia digestiva causada por lesión de Dieulafoy.

Material y métodos: se revisaron de forma retrospectiva todos los casos de hemorragia digestiva por lesión de Dieulafoy entre los años 2000 y 2006. Se recogieron los principales datos clínicos y endoscópicos, tipo de tratamiento empleado, eficacia del mismo, recidiva, complicaciones y mortalidad durante el ingreso.

Resultados: se encontraron 41 pacientes, 26 varones y 15 mujeres, con edad media de 71,19 años. La lesión de Dieulafoy fue la causa del 1,55\% de los casos de hemorragia digestiva aguda en el periodo estudiado. La incidencia de hemorragia digestiva por lesión de Dieulafoy fue de 2,2 casos por cada 100.000 habitantes y año. La mayoría de los pacientes presentaban hemorragia activa en el momento de la endoscopia $(85,36 \%)$ y comorbilidad $(92,68 \%)$. La localización más frecuente fue el estómago $(60,97 \%)$, seguida del duodeno $(29,26 \%)$. El tratamiento endoscópico logró la hemostasia inicial en el 100\% de los casos. Tres pacientes $(7,31 \%)$ presentaron recidiva hemorrágica, todos ellos habían sido tratados inicialmente con esclerosis con adrenalina y respondieron adecuadamente a un segundo tratamiento endoscópico. Ningún paciente precisó cirugía. La mortalidad durante el ingreso fue del $4,87 \%$.

Conclusiones: la lesión de Dieulafoy es una causa poco frecuente, pero potencialmente grave, de hemorragia digestiva y puede aparecer en cualquier punto del tracto gastrointestinal. El tratamiento endoscópico es eficaz y presenta pocas complicaciones. La esclerosis única con adrenalina se asocia a un mayor riesgo de recidiva hemorrágica.

Palabras clave: Dieulafoy. Hemorragia digestiva. Tratamiento endoscópico.

\begin{abstract}
Objective: the aim of the study was to assess the incidence, clinical presentation, location, and response to endoscopic therapy of gastrointestinal bleeding from Dieulafoy's lesion.

Material and methods: all consecutive episodes of gastrointestinal bleeding due to Dieulafoy's lesion seen between 2000 and 2006 were retrospectively reviewed. All main clinical and endoscopic data were collected: type and efectiveness of endoscopic therapy, rebleeding, complications, and mortality during hospitalization.

Results: we found 41 patients, 26 males and 15 females, median age of 71.19 years. Dieulafoy's lesion accounted for $1.55 \%$ of all gastrointestinal bleeding episodes during the study period. The incidence of Dieulafoy's lesion was 2.2 cases/100.000 inhabitants/year. Active bleeding at endoscopy was present in $85.36 \%$, and comorbidity in $92.68 \%$. The stomach was the most frequent location (60.97\%), followed by duodenum (29.26\%). Endoscopic therapy achieved initial hemostasis in all cases. Three patients $(7.31 \%)$ initially treated with epinephrine injection showed rebleeding and properly responded to a second session of endoscopic therapy. No surgery was needed. The mortality rate during hospitalization was $4.87 \%$.

Conclusions: Dieulafoy's lesion is an uncommon, but potentially severe cause of gastrointestinal bleeding. It may be found in any location within the gastrointestinal tract. Endoscopic therapy is effective and safe. Injected epinephrine alone is associated with a higher risk of rebleeding.
\end{abstract}

Key words: Dieulafoy. Gastrointestinal bleeding. Endoscopic therapy.

Ibañez A, Castro E, Fernández E, Baltar R, Vázquez S, Ulla JL, Álvarez V, Soto S, Barrio J, Carpio D, Turnes J, Ledo L, Vázquez San Luis J, Vázquez Astray E. Aspectos clínicos y tratamiento endoscópico de la hemorragia digestiva por lesión de Dieulafoy. Rev Esp Enferm Dig 2007; 99: 505-510.

Recibido: 26-03-07.

Aceptado: 13-05-07.

Correspondencia: Alberto Ibáñez Pinto. C/ Río Umia, 12, 1º. A Caeira, Poio.36005 Pontevedra. e-mail: carthagoo@hotmail.com

\section{INTRODUCCIÓN}

La lesión de Dieulafoy (LD) consiste en un vaso arterial submucoso de calibre persistente que puede ero- 
sionar la mucosa y romperse hacia la luz del tubo digestivo ocasionando hemorragia digestiva a partir de un defecto de la mucosa de mínimo tamaño $(1,2)$. Su localización más frecuente es el estómago alto, en los 6 $\mathrm{cm}$ distales a la unión esófago-gástrica (3). Se han comunicado casos de LD localizados en cualquier punto del tubo digestivo (4-7). La incidencia real de la LD no se conoce bien. Su diagnóstico endoscópico puede ser difícil dado el pequeño tamaño de la lesión y el carácter intermitente de la hemorragia que provoca (8). Varias series recientes estiman que la LD puede ser la causa de hasta el $4-5 \%$ de las hemorragias digestivas altas $(9,10)$.

El tratamiento endoscópico de la LD logra hemostasia en la mayoría de los pacientes, con bajas tasas de recidiva y complicaciones (8-13). Se han empleado diversas técnicas endoscópicas: inyección de adrenalina (IA) y otras sustancias esclerosantes, coagulación con sondas de calor (CSC) o argón y técnicas de índole mecánica como la ligadura endoscópica con bandas (LEB) o la aplicación de hemoclips (8-15). Todas las modalidades de tratamiento endoscópico permiten obtener tasas de hemostasia inicial por encima del $70 \%$, con tasas de recidiva hemorrágica habitualmente menores del 10\% (8-15). En los últimos años se han comunicado buenos resultados tanto con el empleo de hemoclips como con la LEB por lo que estos métodos han sido propuestos como tratamiento de elección (14-18), pero en la práctica la elección del tratamiento depende del criterio y de la experiencia del endoscopista.

El objetivo del presente estudio es conocer la incidencia, la frecuencia de aparición respecto al total de casos de hemorragia digestiva, forma de presentación, localización y resultados de las diversas técnicas de tratamiento endoscópico en la hemorragia digestiva causada por LD.

\section{MATERIAL Y MÉTODOS}

Se revisaron de forma retrospectiva todos los casos de hemorragia digestiva por LD diagnosticados entre el 1 de enero de 2000 y el 31 de diciembre de 2006. Se recogió el número total de pacientes con hemorragia digestiva aguda (HDA) atendidos en la Unidad de Endoscopia en el mismo periodo. Los criterios diagnósticos de LD empleados fueron los descritos previamente en la literatura $(9,15,19)$ : a) hemorragia arterial en chorro o sangrado babeante micropulsátil procedente de un defecto mucoso mínimo, rodeado de mucosa normal; b) vaso protruyente sobre un defecto mucoso mínimo, rodeado de mucosa normal, con o sin hemorragia activa; o c) presencia de un coágulo fresco adherido sobre un defecto mínimo mucoso o sobre mucosa normal. Todos los pacientes o sus familiares directos firmaron previamente la hoja de consentimiento informado. Las endoscopias fueron realizadas en todos los casos por especia- listas en aparato digestivo con experiencia en endoscopia diagnóstica y terapéutica. Antes de la endoscopia se obtuvo un hemograma y un estudio básico de coagulación y todos los pacientes recibieron tratamiento de soporte para garantizar su estabilidad hemodinámica en el área de urgencias, hospitalización o en la Unidad de Cuidados Intensivos. Los enfermos con coagulopatía o bajo tratamiento anticoagulante recibieron vitamina $\mathrm{K}$ o plasma fresco congelado. Los pacientes sometidos a colonoscopia fueron preparados con Fosfosoda ${ }^{\circledR}$ (Laboratorio Casen Fleet), solución evacuante Bohm ${ }^{\circledR}$ (Laboratorio Bohm) y/o enemas de limpieza. Las gastroscopias fueron practicadas con videoendoscopios Olympus GIF-IT140, GIF-XP160 y GIFV2, las colonoscopias con videoendoscopios Olympus CF-Q145L y el modelo de enteroscopio utilizado fue Olympus SIF-100.

En todos los casos la terapéutica endoscópica se realizó en el mismo momento del diagnóstico. La modalidad de tratamiento empleada fue elegida según el criterio del endoscopista en cada caso. Para la esclerosis endoscópica se emplearon agujas tipo Boston Scientific modelo 1123, de $1,8 \mathrm{~mm}$ de ancho y $23 \mathrm{GA}$ de calibre. Las soluciones empleadas para la esclerosis fueron: adrenalina 1/10.000, polidocanol al $2 \%$ y oleato de etanolamina al $5 \%$. Los hemoclips utilizados fueron Olympus HX-201UR-135. El plasma de argón se empleó mediante sonda Olympus MAJ-1012 a volúmenes de entre 1,5-1,7 1/min y con potencia variable entre 40 y $50 \mathrm{~W}$. La hemostasia inicial se definió como cese de la hemorragia al terminar la endoscopia o bien destrucción, desaparición o taponamiento del vaso visible. Se definió la recidiva como la presencia de clínica hemorrágica (hematemesis, melena, rectorragia, hematoquecia o hipotensión) junto con el hallazgo de coágulo fresco adherido o sangrado activo a partir de la misma lesión y en la misma localización encontrada en la endoscopia inicial.

Los datos clínicos se obtuvieron de las siguientes fuentes: a) la base de datos médicos e informes clínicos, VisHC, propia del centro; b) el programa de historia clínica digital IANUS; y c) el archivo de historias clínicas en formato papel. Los datos sobre las exploraciones endoscópicas se obtuvieron a partir de la base de datos de la unidad de endoscopia, ENDOBASE y a partir del archivo de informes de endoscopia en formato papel. El análisis estadístico de los datos se realizó mediante el programa informático Excel ${ }^{\circledR}$.

\section{RESULTADOS}

Durante el periodo estudiado se diagnosticaron 41 casos de HDA por LD, 26 varones y 15 mujeres, con edades comprendidas entre 24 y 96 años. La edad media fue de 71,19 años. En el mismo periodo se atendieron 2.645 pacientes por HDA. La LD fue la causa de la hemorragia en el $1,55 \%$ de los casos. Dos pacientes fueron remitidos desde otro centro para la realización de una enteroscopia. 
La incidencia calculada de hemorragia digestiva por LD, en nuestra área sanitaria es de 2,2 casos por cada 100.000 habitantes y año.

Treinta y ocho pacientes $(92,68 \%)$ presentaban al menos una enfermedad crónica sistémica y $34(82,92 \%)$ dos o más causas de comorbilidad. Las enfermedades crónicas de los pacientes quedan expuestas en la tabla I. Veinte pacientes $(48,78 \%)$ estaban en el momento de la hemorragia bajo tratamiento con anticoagulantes, antiagregantes o antiinflamatorios no esteroideos (AINE). Cinco con anticoagulantes orales $(12,95 \%), 15$ con AINE o antiagregantes $(36,58 \%)$ y un paciente estaba antiagregado y anticoagulado.

La forma de presentación clínica fue: melenas en 19 pacientes $(46,34 \%)$, hematemesis en $13(31,70 \%)$, hematoquecia en $5(12,19 \%)$, rectorragia en $3(7,31 \%)$ e hipotensión sin signos externos de hemorragia en $1(2,43 \%)$. Doce pacientes $(29,26 \%)$ presentaban inestabilidad hemodinámica, definida como tensión arterial sistólica menor de $100 \mathrm{mmHg}$ en la exploración física inicial. La hemoglobina inicial estaba en un rango de 4,5-14,5 g/dl, con una media de $8,5 \mathrm{~g} / \mathrm{dl}$. Treinta cinco pacientes $(85,36 \%)$ necesitaron transfusión de concentrados de hematíes durante el ingreso, con una media de 3,12 concentrados (rango 0-8 concentrados de hematíes) por paciente.

El número medio de exploraciones endoscópicas necesarias para localizar la lesión sangrante fue de 1,43, con rango comprendido entre 1 y 4 . Doce pacientes $(29,26 \%)$ precisaron más de una endoscopia para el diagnóstico. Dos pacientes necesitaron 4 exploraciones, ambos sangraban a partir de lesiones situadas en yeyuno y duodeno distal y fueron diagnosticados mediante enteroscopia tras la realización de gastroscopia y colonoscopia. Treinta y cinco pacientes $(85,36 \%)$ presentaban hemorragia activa en el momento de la endoscopia. La localización más frecuente fue el estómago $(60,97 \%)$, seguida del duodeno $(29,26 \%)$. La localización de las lesiones queda detallada en la tabla II. De los 12 pacientes con LD duodenal, en 3 la lesión se encontraba en el duodeno distal, fuera del alcance del gastroscopio y fue necesaria una enteroscopia para su diagnóstico.

Se emplearon cinco modalidades de tratamiento endoscópico sobre un total de 44 tratamientos: a) IA en 11 casos (25\%); b) IA más un segundo esclerosante (polidocanol o etanolamina) en 10 pacientes $(22,72 \%)$; c) IA más electrofulguración con argón en $18(40,90 \%)$; d) IA más hemoclips en $3(6,8 \%)$ y e) electrofulguración sólo con argón en $2(4,54 \%)$. Se logró hemostasia inicial en todos los casos. Se detectaron 3 recidivas hemorrágicas $(7,31 \%)$ a los 15 días, 7 y 11 meses. Las tres lesiones que recidivaron habían sido tratadas inicialmente con IA. La tasa de recidiva de la IA es del 27,27\%. Las recidivas fueron tratadas endoscópicamente mediante esclerosis más argón en dos casos y esclerosis más hemoclips en 1, con buen resultado. Ningún paciente precisó tratamiento quirúrgico. Un paciente tratado inicialmente con
Tabla I. Causas de comorbilidad en los pacientes con LD

\begin{tabular}{lc}
\hline Causas de comorbilidad & Número pacientes \\
\hline Cardiopatía valvular & 8 \\
Cardiopatía isquémica & 8 \\
Insuf. cardiaca de otro origen & 3 \\
Insuficiencia renal o trasplante renal & 15 \\
Cirrosis-hepatopatía crónica & 6 \\
EPOC & 11 \\
HTA & 19 \\
DM & 9 \\
Arteriopatía periférica & 3 \\
Enfermedad cerebro-vascular & 3 \\
Obesidad mórbida & 1 \\
Hipotiroidismo & 1 \\
Leucemia aguda-linfoma & 2 \\
Cáncer sólido & 2 \\
Hepatocarcinoma & 1 \\
Ca. próstata & 1 \\
Fa crónica & 4 \\
Insuficiencia suprarrenal & 1 \\
\hline
\end{tabular}

Tabla II. Localización de las LD

\begin{tabular}{lc}
\hline Localización & Número de casos \\
\hline Estómago & $25(60,97 \%)$ \\
Cardias, fundus, zona subcardial o cuerpo alto & $16(39,2 \%)$ \\
Cuerpo bajo, incisura o antro & $9(21,95 \%)$ \\
Duodeno & $12(29,26 \%)$ \\
Yeyuno & $1(2,43 \%)$ \\
Colon & $2(4,87 \%)$ \\
Recto & $1(2,43 \%)$ \\
\hline
\end{tabular}

Tabla III. Otras lesiones encontradas en la endoscopia en pacientes con LD

\begin{tabular}{lc}
\hline Otras lesiones & Número de casos \\
\hline Angiodisplasias & $10(24,39 \%)$ \\
Hernia de hiato & $9(21,95 \%)$ \\
Varices esofágicas & $3(7,31 \%)$ \\
Esofagitis péptica & $2(4,87 \%)$ \\
Duodenitis erosiva & $1(2,43 \%)$ \\
LAMG & $1(2,43 \%)$ \\
Pólipos & $2(4,87 \%)$ \\
Tumor submucoso & $1(2,43 \%)$ \\
Esofagitis candidiásica & $1(2,43 \%)$ \\
\hline
\end{tabular}

argón en el duodeno presentó, 48 horas después, una perforación puntiforme y cubierta que se resolvió adecuadamente mediante sutura endoscópica con clips, antibioterapia y nutrición parenteral. No se detectaron otras complicaciones secundarias a la terapia endoscópica. Trece pacientes presentaron complicaciones sistémicas $(31,70 \%)$, principalmente asociadas a un empeoramiento de sus enfermedades previas. Dos pacientes fallecieron durante el ingreso (tasa de mortalidad del $4,87 \%$ ) por insuficiencia cardiorrespiratoria en un caso y fracaso multiorgánico en el otro. 


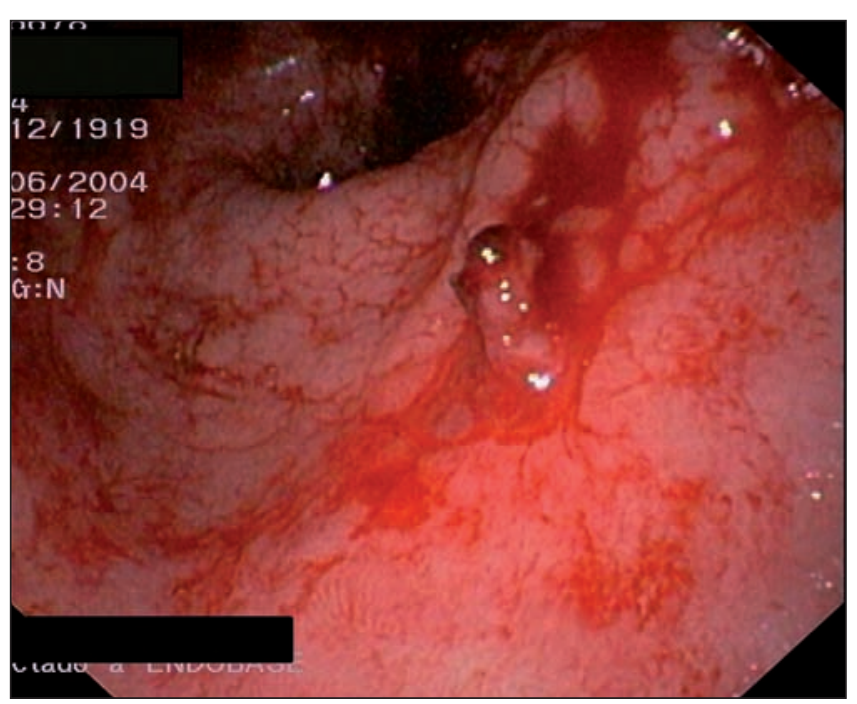

Fig. 1. Lesión de Dieulafoy en el antro gástrico. Se aprecia un vaso arterial prominente, rodeado de mucosa normal.

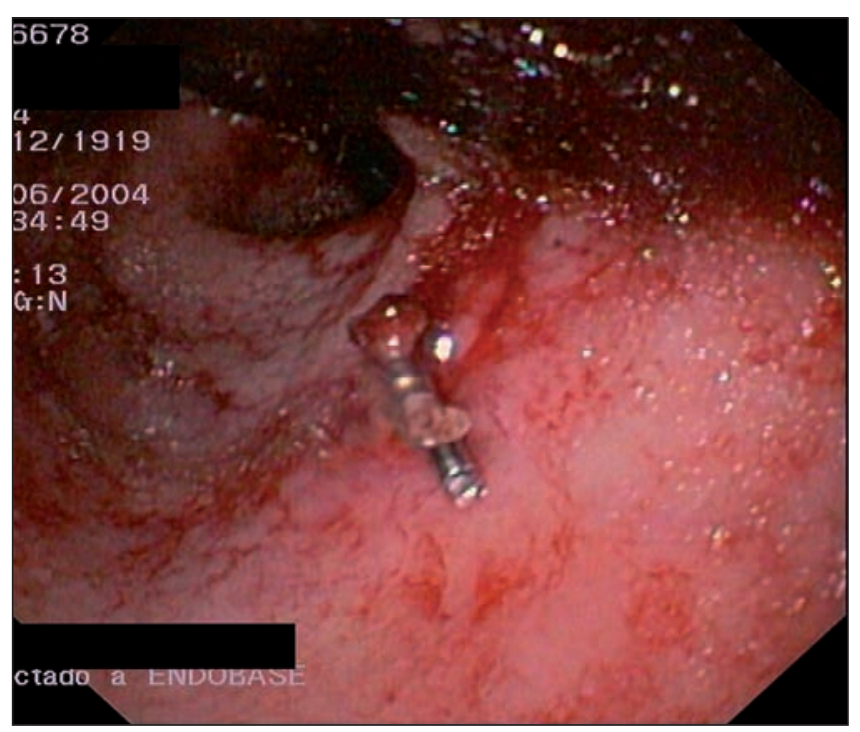

Fig. 2. La misma lesión tras la aplicación de dos hemoclips que permiten taponar el vaso.

La endoscopia detectó otras lesiones no causantes de la hemorragia en 21 pacientes $(51,21 \%)$ (Tabla III). Diez pacientes $(24,39 \%)$ presentaban angiodisplasias que estaban localizadas en otros tramos del tubo digestivo, alejadas del punto sangrante y no mostraban signos de hemostasia reciente en ningún caso.

\section{DISCUSIÓN}

Descrita inicialmente como una causa rara de HDA, la LD es reconocida cada vez con más frecuencia como una causa relevante de hemorragia gastrointestinal $(9,10)$. En nuestro estudio, la incidencia de la LD es de 2,2 casos por cada 100.000 habitantes y año y representa el $1,55 \%$ de todas las causas de HDA, tanto de origen alto como bajo. Estos resultados son comparables a los descritos en otras series $(12,14)$ y señalan que la $\mathrm{LD}$, aunque infrecuente, no es una patología excepcional.

Los datos clínicos y epidemiológicos de los pacientes analizados coinciden con los descritos en la literatura $(8,12,20)$. La LD aparece preferentemente en pacientes de edad avanzada y con comorbilidad. En nuestra serie el $92,68 \%$ de los enfermos presentaban al menos una enfermedad crónica sistémica y el $82,92 \%$ dos o más causas de comorbilidad (Tabla I). Se desconoce cuál es el mecanismo que puede condicionar la aparición de LD en este tipo de pacientes. Es bien conocida la asociación entre enfermedades sistémicas como la cirrosis hepática y la IRC y las lesiones vasculares en el tracto digestivo como las angiodisplasias o la ectasia vascular antral $(21,22)$. Posiblemente este tipo de trastornos sistémicos alteren la angiogénesis normal y condicionen la aparición de neovasos arteriales aberrantes de calibre persistente que, en condiciones de estrés de la mucosa digestiva, pueden erosionarse y provocar hemorragia. Es previsible que el envejecimiento de la población junto con una mayor prevalencia de este tipo de enfermedades crónicas hagan aumentar la frecuencia de HDA causada por lesiones vasculares, incluida la LD.

El 48,78\% de los enfermos de nuestro estudio se hallaban bajo tratamiento con anticoagulantes orales, antiagregantes o AINE. Probablemente este dato está relacionado con el elevado número de pacientes con enfermedad o factores de riesgo vascular de nuestra serie. Similares porcentajes han sido descritos en la literatura (8-12). No es posible descartar que este tipo de fármacos puedan contribuir en la erosión y rotura de la $\mathrm{LD}$, tal y como ha sido sugerido por algunos autores previamente (1).

En nuestro estudio, un 29,26\% de los pacientes presentaban inicialmente inestabilidad hemodinámica y un $85,36 \%$ mostraban hemorragia activa en el momento de la endoscopia. La mayoría necesitaron transfusión de hemoderivados. En doce enfermos la LD no fue diagnosticada en la primera endoscopia y necesitaron ulteriores exploraciones para localizar el punto sangrante. Estos datos ponen de manifiesto que la LD es una causa potencialmente grave de HDA que en ocasiones puede ser de difícil diagnóstico. Es necesario un alto índice de sospecha y una exploración exhaustiva del tracto gastrointestinal que permita un diagnóstico y un tratamiento precoz.

La LD puede aparecer en cualquier punto del tracto gastrointestinal y ser causa de hemorragia digestiva tanto alta como baja. En nuestra serie, la localización más frecuente es el estómago $(60,97 \%)$, seguida del duodeno $(29,26 \%)$. Además destacan cuatro casos situados en el duodeno distal-yeyuno que precisaron de una enteroscopia para el diagnóstico, dos casos en el colon y un caso en el recto. La localización de las lesiones coincide con los 
datos de otras series (20) y con las descripciones de la LD en puntos infrecuentes como el esófago, intestino delgado o el recto (4-6).

Se ha empleado una amplia variedad de técnicas de hemostasia endoscópica en la LD $(9-18,20)$. Sólo existen dos estudios prospectivos y aleatorizados que hayan comparado la eficacia de dos técnicas de tratamiento endoscópico $(16,17)$. Chung y cols. compararon los resultados del tratamiento con inyección de suero salino hipertónico (SSH) frente al taponamiento mecánico con hemoclips o LEB en una serie de 24 pacientes. La eficacia a la hora de lograr la hemostasia inicial fue mayor en el grupo de tratamiento mecánico mientras que la tasa de recidiva hemorrágica fue significativamente mayor en el grupo tratado con SSH (16). En el segundo estudio, Park y cols. no observaron diferencias estadísticamente significativas en las tasas de hemostasia inicial al comparar la IA frente al tratamiento con hemoclips, pero este último tratamiento sí fue significativamente más eficaz a la hora de prevenir la recurrencia hemorrágica (17). Cheng y cols. observan también una tasa de hemostasia significativamente mayor en el tratamiento con CSC en comparación con la IA, aunque este estudio no es prospectivo (23). Otros autores han comunicado también resultados mejores al comparar retrospectivamente el tratamiento con CSC frente a la inyección de sustancias esclerosantes (13). En los últimos años se han publicado varios trabajos retrospectivos que muestran buenos resultados en el control inicial de la hemorragia y bajas tasas de recidiva tanto con el empleo de hemoclips como con la LEB, lo que probablemente indica una tendencia hacia un mejor resultado con este tipo de métodos en comparación con la inyección de sustancias esclerosantes (14-18).

Los pacientes de nuestro estudio fueron tratados endoscópicamente mediante cinco modalidades distintas de tratamiento. La elección de la técnica endoscópica dependió del criterio del endoscopista en cada caso. Probablemente esta práctica refleja la forma habitual de enfrentarse a la LD en la mayoría de las unidades de endoscopia. El tratamiento más empleado fue la IA combinada con electrofulguración con argón. También empleamos con frecuencia la IA única y la IA combinada con polidocanol o etanolamina. Utilizamos hemoclips combinados con IA en un 6,8\% y la coagulación solo con argón en un 4,54\%. Se logró la hemostasia inicial en el $100 \%$ de los casos, con una tasa de recidiva de tan sólo un 7,31\%. Ningún paciente precisó tratamiento quirúrgico. Los tres casos de recidiva hemorrágica acontecieron en pacientes que habían sido tratados sólo con IA, lo que traduce una tasa de recidiva para este tipo de tratamiento del $27,7 \%$ frente al $0 \%$ del resto de las técnicas.

Schmulewitz y cols. sugieren que la vasoconstricción local inducida por la IA puede provocar una hemostasia inicial aparente, pero sería insuficiente a la hora de conseguir una trombosis total en el vaso arterial de gran calibre que caracteriza a la LD (20). Este fenómeno explica- ría la aparición de recidivas tardías como ocurrió en dos de nuestros pacientes.

Probablemente los tratamientos con CSC, argón, hemoclips y LEB son más eficaces a la hora de lograr una destrucción o trombosis total de la LD. Sin embargo estos métodos son técnicamente difíciles de aplicar en las localizaciones donde la LD es más frecuente, como la zona subcardial y el estómago alto, especialmente en casos de hemorragia activa de gran débito. El tratamiento combinado mediante IA, seguido de un tratamiento de índole mecánico o la coagulación con argón es una opción razonable que permite controlar inicialmente la hemorragia, mejorando la visión de la lesión y haciendo técnicamente más sencilla y segura la aplicación del segundo tratamiento endoscópico.

Son necesarios más estudios que permitan definir con precisión cuál es la modalidad de tratamiento endoscópico más apropiada en la LD.

\section{BIBLIOGRAFÍA}

1. Juler GL, Labitzke HG, Lamb R, Allen R. The pathogenesis of Dieulafoy's gastric erosion. Am J Gastroenterol 1984; 79: 195-200.

2. Tejero Cebrian E, García Aguilar J, Arias Pérez J, Alia Benítez J, Jorge Sánchez E, Durán Sacristán H. Enfermedad de Dieulafoy: una causa rara de hemorragia digestiva alta. Rev Esp Enferm Dig 1987; 72: 171-2.

3. Veldhuyzen van Zanten SJ, Bartelsman JF, Schipper ME, Tytgat GN Recurrent massive haematemesis from Dieulafoy vascular malformations-a review of 101 cases. Gut 1984; 79: 195-200.

4. Scheider DM, Barthel JS, King PD, Beale GD. Dieulafoy like lesion of the distal esophagus. Am J Gastroenterol 1994; 89: 2080-1.

5. Meister TE, Varilek GW, Marsano LS, Gates LK, Al-Tawil Y, de Villiers WJ. Endoscopic management of rectal Dieulafoy like lesions: A case series and review of literature. Gastrointest Endosc 1998; 48: $302-5$.

6. Sone Y, Nakano S, Takeda I, Kumada T, Kiriyama S, Hisanaga Y. Massive hemorrhage from a Dieulafoy lesion in the cecum: Successful endoscopic management. Gastrointest Endosc 2000; 51: 510-2.

7. Blecker D, Bansal M, Zimmerman RL, Fogt F, Lewis J, Stein R, et al Dieulafoy's lesion of the small bowel causing massive gastrointestinal bleeding: two case reports and literature review. Am J Gastroenterol 2001; 96: 902-5.

8. Baetting B, Haecki W, Lammer F, Jost R. Dieulafoy's disease: Endoscopic treatment and follow up. Gut 1993; 34: 1418-21.

9. Sone Y, Kumada T, Toyoda H, Hisanaga Y, Kiriyama S, Tanikawa $\mathrm{M}$. Endoscopic management and follow up of Dieulafoy lesion in the upper gastrointestinal tract. Endoscopy 2005; 37: 449-53.

10. Romaozinho JM, Pontes JM, Lérias C, Ferreira M, Freitas D. Dieulafoy's lesion: Management and long term outcome. Endoscopy 2004; 36: 416-20.

11. Pointner R, Schwab G, Konigsrainer A, Dietze O. Endoscopic treatment of Dieulafoy's disease. Gastroenterol 1988; 94: 563-6.

12. Norton ID, Petersen BT, Sorbi D, Balm RK, Alexander GL, Gostout CJ. Management and long term prognosis of Dieulafoy lesion. Gastrointest Endosc 1999; 50: 762-7.

13. Kasapidis P, Georgopoulos P, Delis V, Balastos V, Konstantinidis A, Skandalis N. Endoscopic management and long term folow up of Dieulafoy's lesion in the upper GI tract. Gastrointest Endosc 2002; 55: 527-31.

14. Yamaguchi Y, Yamato T, Katsume N, Imao Y, Aoki K, Morita Y, et al. Short-term and long-term benefits of endoscopic hemoclip application for Dieulafoy's lesion in the upper GI tract. Gastrointest Endosc 2003; 57: 653-6.

15. Nikolaidis N, Zezos P, Giouleme O, Budas K, Marakis G, Paroutoglou G, et al. Endoscopic band ligation of Dieulafoy-like lesions in 
the upper gastrointestinal tract. Endoscopy 2001; 33: 754-60.

16. Park CH, Sohn YH, Lee WS, Joo YE, Choi SK, Rew JS, et al. The usefulness of endoscopic hemoclipping for bleeding Dieulafoy lesions. Endoscopy 2003; 35: 388-92.

17. Chung IK, Kim EJ, Lee MS, Kim HS, Park SH, Lee MH, et al. Bleeding Dieulafoy's lesions and the choice of endoscopic method: comparing the hemostatic efficacy of mechanical and injection methods. Gastrointest Endosc 2000; 52: 721-4.

18. Parra Blanco A, Takahashi H, Méndez Jerez PV, Kojima T, Aksoz K, Kirihara K, et al. Endoscopic management of Dieulafoy lesions of the stomach: A case study of 26 patients. Endoscopy 1997; 29: 834-9.

19. Dy NM, Gostout CJ, Balm RK. Bleeding from endoscopically-identified Dieulafoy lesion of the proximal small intestine and colon. Am J
Gastroenterol 1995; 90: 108-11.

20. Schmulewitz N, Baillie J. Dieulafoy lesions: A review of 6 years of experience at a tertiary referral center. Am J Gastroenterol 2001; 96 : 1688-94.

21. Chalasani N, Cotsonis G, Wilcox CM. Upper gastrointestinal bleeding in patient with chronic renal failure: Role of vascular ectasia. Am J Gastroenterol 1996; 91: 2329-32.

22. Burak KW, Lee SS, Beck PL. Portal Hypertensive gastropathy and gastric antral vascular ectasia (GAVE) syndrome. Gut 2001; 49: 86672.

23. Cheng CL, Liu NJ, Lee CS, Chen PC, Ho YP, Tang JH, et al. Endoscopic management of Dieulafoy lesions in acute nonvariceal upper gastrointestinal bleeding. Dig Dis Sci 2004; 49: 1139-44. 